Article

\title{
Phase Inversion in PVDF Films with Enhanced Piezoresponse Through Spin-Coating and Quenching
}

\author{
Marco Fortunato 1,2,*, Domenico Cavallini ${ }^{1,2}$, Giovanni De Bellis ${ }^{1,2}$, Fabrizio Marra ${ }^{1,2}$, \\ Alessio Tamburrano ${ }^{1,2}$, Francesca Sarto $^{3}$ and Maria Sabrina Sarto ${ }^{1,2}$ (D) \\ 1 Department of Astronautical, Electrical and Energy Engineering, Sapienza University of Rome, \\ Via Eudossiana 18, Rome 00184, Italy \\ 2 Research Center for Nanotechnology Applied to Engineering of Sapienza (CNIS), SNNLab, \\ Sapienza University of Rome, Piazzale Aldo Moro, 5, Rome 00185, Italy \\ 3 ENEA, Frascati Research Center, Via Enrico Fermi, 45, Frascati 00044, Italy \\ * Correspondence: marco.fortunato@uniroma1.it; Tel.: +39-06-4458-5528
}

Received: 16 May 2019; Accepted: 24 June 2019; Published: 28 June 2019

\begin{abstract}
In the present work, poly(vinylidene fluoride) (PVDF) films were produced by spin-coating, and applying different conditions of quenching, in order to investigate the dominant mechanism of the $\beta$-phase formation. The influence of the polymer/solvent mass ratio of the solution, the rotational speed of the spin-coater and the crystallization temperature of the film on both the $\beta$-phase content and the piezoelectric coefficient $\left(d_{33}\right)$ were investigated. This study demonstrates that the highest values of $d_{33}$ are obtained when thinner films, produced with a lower concentration of polymer in the solvent (i.e., $20 \mathrm{wt}$ \%), go through quenching in water, at room temperature. Whereas, in the case of higher polymer concentration (i.e., $30 \mathrm{wt} . \%)$, the best value of $d_{33}(\sim 30 \mathrm{pm} / \mathrm{V})$ was obtained through quenching in liquid nitrogen, at the temperature of $77 \mathrm{~K}$. We believe that in the former case, phase inversion is mainly originated by electrostatic interaction of PVDF with the polar molecules of water, due to the low viscosity of the polymer solution. On the contrary, in the latter case, due to higher viscosity of the solution, mechanical stretching induced on the polymer during spin-coating deposition is the main factor inducing self-alignment of the $\beta$-phase. These findings open up a new way to realize highly efficient devices for energy harvesting and wearable sensors.
\end{abstract}

Keywords: polyvinylidene fluoride (PVDF); quenching; piezoelectric effect; piezoresponse force microscopy (PFM)

\section{Introduction}

In the last ten years, piezoelectric polymer thin films have attracted a lot of interest in the production of flexible nanogenerators, sensors, and actuators [1-4]. Poly(vinylidene fluoride) (PVDF) is one of the most interesting piezoelectric polymers for a wide range of advanced applications, from sensing to energy harvesting $[1,5]$. The great interest in PVDF is due to its excellent thermal stability, mechanical flexibility, low-density and unique piezoelectric and ferroelectric characteristics. PVDF exists in three main polymorphs phases $(\alpha, \beta$, and $\gamma)$. The most common crystalline phases in PVDF are the $\alpha$ - and the $\beta$-phase. The $\alpha$-phase is an electrically inactive phase, whereas the $\beta$-phase is an electroactive and polar phase, showing the strongest ferroelectric and piezoelectric behaviour [6]. In order to increase the electroactive response of PVDF, the $\mathrm{CH}_{2}-\mathrm{CF}_{2}$ dipoles must be aligned and oriented along a preferential direction. Dipoles orientation is usually obtained by electrical poling. This technique is based on the application of a strong DC electric field $\left(\sim 10^{6} \mathrm{~V} / \mathrm{m}\right)$ at elevated temperatures $(\sim 393 \mathrm{~K})$ on the polymer through a top and bottom electrode, in order to induce the alignment of the dipoles along the direction of the electric field [7]. However, electrical poling has some limitations in terms 
of cost-effectiveness and practical implementation. Recently, several alternative techniques, aimed at improving the nucleation of the electroactive state in PVDF, such as mechanical stretching, heat controlled spin-coating, and phase inversion, have been investigated [8-10]. In particular the phase inversion technique was investigated in Reference [10], and the effect of temperature on the piezoelectric coefficient was examined in the $253 \mathrm{~K}-373 \mathrm{~K}$ range. It was demonstrated that at the temperature of 253 $\mathrm{K}$, the $\beta$-phase crystallites are self-aligned, and the PDVF film is characterized by a high piezoelectric coefficient. It resulted that self-aligned $\beta$-phase PVDF films with high piezoelectric coefficient were produced via phase inversion, through spin-coating and quenching. However, the mechanism for the self-alignment of the $\beta$-crystals was not fully understood, due to the competitive role of three different factors: The mechanical stretching of the polymer chains during spinning, the thermal gradient during quenching, the electrostatic interaction between the polymer film and the polar molecules of water.

In References [2,11], the authors investigated the influence on the $\beta$-phase formation and the piezoelectric response enhancement of several process parameters. These included the polymer concentration (from 10 to $24 \mathrm{wt} . \%$ ), the rotational speed ranging from $1000 \mathrm{rpm}$ to $8000 \mathrm{rpm}$, and the crystallization temperature, in the range from $293 \mathrm{~K}$ to $363 \mathrm{~K}$. It was shown that the $\beta$-phase increases in general with the rotation speed of the spin-coater, up to a limit value, which increases with the polymer concentration in the solvent. Moreover, the electroactive phase reduces dramatically as the baking temperature increases over $323 \mathrm{~K}$.

The scope of this work is to assess to what extent the mechanical stretching of the polymeric chains, produced by the spin-coater rotation during film deposition, is responsible for the formation of the $\beta$-phase and the enhancement of the piezoelectric response.

For this purpose, piezoelectric PVDF thin films were produced through spin-coating and phase inversion by quenching, avoiding the use of electrical poling. For the first time, we investigated the effect of quenching on spin-coated PVDF films at very low temperature $(\sim 77 \mathrm{~K})$ in a non-polar medium (i.e., liquid nitrogen) in order to avoid polarization, due to electrostatic interaction or temperature gradient. In particular, we studied how the piezoelectric performance of PVDF films is affected by: (i) The polymer/solvent mass ratio; (ii) the rotational spin speed; and (iii) the quenching temperature. The relative volume fraction of the $\beta$-phase $(F(\beta))$ of the produced films was evaluated through Fourier Transform Infrared (FT-IR) spectroscopy, and the piezoelectric response was assessed through measurements of the coefficient $d_{33}$ using Piezoresponse Force Microscopy (PFM). We quantitatively evaluated the $d_{33}$ avoiding the use of a top electrode [1] and using the characterization protocol based on PFM described in Reference [12]. The morphology of the sample was evaluated through Field Emission Scanning Electron Microscopy (FE-SEM). The viscosity of the prepared PVDF solutions was measured, before film deposition, through rheology. The obtained results demonstrated that, based on the viscosity of the PVDF solution and on the quenching temperature, the best piezoelectric response is originated either by the electrostatic interaction of the polymer chains with polar molecules of water or by the mechanical stretching induced by the spin-coater's rotation.

\section{Materials and Methods}

The PVDF samples were obtained by the combination of spin-coating and quenching treatment in order to increase the piezoelectric coefficient $d_{33}$ and the electroactive phase $F(\beta)$ content.

The samples were produced as follows: firstly, PVDF in powder form (Solvay 6010, Solvay Specialty Polymers S.P.A., Bollate, Italy) was dissolved in N,N dimethylformamide (DMF $\geq 99 \%$, Sigma-Aldrich, Saint Louis, MO, USA) by magnetic stirring (Heidolph Instruments, Schwabach, Germany) for $3 \mathrm{~h}$ at $343 \mathrm{~K}$. Then, $1 \mathrm{~mL}$ of the produced polymer solution was cast over a PET/ITO substrate $\left(R_{\mathrm{s}}=60 \Omega / \mathrm{sq}\right.$., Sigma-Aldrich, Saint Louis, MO, USA) $\left[\sim(3 \times 3) \mathrm{cm}^{2}\right]$ and spin-coated (Semiconductor Production Systems Europe, Putten, Netherlands) with an acceleration of $7500 \mathrm{rpm} / \mathrm{s}^{2}$ for $10 \mathrm{~s}$. After the deposition the sample was immersed immediately in an opportune bath $\left(\mathrm{H}_{2} \mathrm{O}\right.$; $\mathrm{H}_{2} \mathrm{O}+$ glycerol or liquid $\mathrm{N}_{2}$ ) held at various quenching temperatures $(303 \mathrm{~K}, 253 \mathrm{~K}$ or $77 \mathrm{~K})$ for $30 \mathrm{~min}$. 
In this work, we considered the two polymer concentration of $20 \mathrm{wt} \%$ and $30 \mathrm{wt} \%$ Moreover, the films were produced at two different rotation speeds of the spin-coater (i.e $2500 \mathrm{rpm}$ and $7500 \mathrm{rpm}$ ) and considering the following three quenching conditions-(i) $303 \mathrm{~K}$ in a water bath; (ii) $253 \mathrm{~K}$ in an ice/glycerol bath; and (iii) $77 \mathrm{~K}$ in a liquid nitrogen bath.

The produced samples are schematically summarized in Table 1 . The films produced with $20 \mathrm{wt} . \%$ of PVDF at $2500 \mathrm{rpm}$ or $7500 \mathrm{rpm}$ have average thicknesses of approximately $(17 \pm 3) \mu \mathrm{m}$ or $(7.7 \pm 1.5)$ $\mu \mathrm{m}$, respectively. For the sample containing $30 \mathrm{wt}$ \% of PDVF, the average thickness is $(46 \pm 2) \mu \mathrm{m}$ or $(20 \pm 2) \mu \mathrm{m}$, corresponding to a spinning speed of $2500 \mathrm{rpm}$ or $7500 \mathrm{rpm}$, respectively.

Table 1. List of the produced specimens of Poly(vinylidene fluoride) (PVDF) thin films, production conditions and piezoelectric properties.

\begin{tabular}{|c|c|c|c|c|c|c|c|c|}
\hline Sample & $\begin{array}{l}\text { PVDF } \\
\text { Concentr. } \\
\text { (wt. \%) }\end{array}$ & $\begin{array}{l}\text { Spinning } \\
\text { Speed } \\
\text { (rpm) }\end{array}$ & $\begin{array}{c}\text { Quenching } \\
\text { Temperature } \\
\text { (K) }\end{array}$ & $\begin{array}{l}\text { Quenching } \\
\text { Medium }\end{array}$ & $\begin{array}{l}\text { Spherulite } \\
\text { Diameter } \\
(\mathrm{nm})\end{array}$ & $\begin{array}{l}F(\beta) \\
(\%)\end{array}$ & $\begin{array}{c}\left\langle d_{33}\right\rangle \\
(\mathrm{pm} / \mathrm{V})\end{array}$ & $\begin{array}{c}d_{33 \max } \\
(\mathrm{pm} / \mathrm{V})\end{array}$ \\
\hline $\mathrm{A}$ & 20 & 7500 & 303 & $\mathrm{H}_{2} \mathrm{O}$ & $754 \pm 110$ & 80 & $15.55 \pm 1.32$ & 16.87 \\
\hline B & 20 & 7500 & 253 & $\mathrm{H}_{2} \mathrm{O}+$ glycerol & - & 73 & $9.92 \pm 0.87$ & 10.79 \\
\hline C & 20 & 7500 & 77 & $\mathrm{~N}_{2}$ & - & 77 & $6.16 \pm 0.41$ & 6.57 \\
\hline $\mathrm{D}$ & 20 & 2500 & 303 & $\mathrm{H}_{2} \mathrm{O}$ & $761 \pm 130$ & 53 & $17.47 \pm 3.56$ & 21.03 \\
\hline $\mathrm{E}$ & 20 & 2500 & 253 & $\mathrm{H}_{2} \mathrm{O}+$ glycerol & - & 79 & $6.63 \pm 0.22$ & 6.85 \\
\hline $\mathrm{F}$ & 20 & 2500 & 77 & $\mathrm{~N}_{2}$ & $268 \pm 44$ & 75 & $7.58 \pm 0.67$ & 8.25 \\
\hline G & 30 & 7500 & 303 & $\mathrm{H}_{2} \mathrm{O}$ & $609 \pm 89$ & 81 & $6.17 \pm 2.83$ & 9.00 \\
\hline $\mathrm{H}$ & 30 & 7500 & 253 & $\mathrm{H}_{2} \mathrm{O}+$ glycerol & $804 \pm 137$ & 80 & $3.99 \pm 0.85$ & 4.84 \\
\hline I & 30 & 7500 & 77 & $\mathrm{~N}_{2}$ & $575 \pm 90$ & 81 & $17.22 \pm 6.24$ & 23.46 \\
\hline $\mathrm{L}$ & 30 & 2500 & 303 & $\mathrm{H}_{2} \mathrm{O}$ & - & 67 & $9.17 \pm 0.52$ & 9.69 \\
\hline $\mathrm{M}$ & 30 & 2500 & 253 & $\mathrm{H}_{2} \mathrm{O}+$ glycerol & $609 \pm 116$ & 74 & $5.47 \pm 2.06$ & 7.53 \\
\hline $\mathrm{N}$ & 30 & 2500 & 77 & $\mathrm{~N}_{2}$ & $386 \pm 61$ & 98 & $21.64 \pm 8.23$ & 29.87 \\
\hline
\end{tabular}

\section{Characterization}

A Field Emission Scanning Electron Microscope (FE-SEM, Auriga, Carl Zeiss, Oberkochen, Germany) operating with an accelerating voltage of $3 \mathrm{kV}$ was used to assess the morphology of the PVDF films. A Quorum Technologies Q150T ES sputter coater (Laughton, East Sussex, UK) was used to metallize the PVDF films prior to SEM imaging with $20 \mathrm{~nm}$ of $\mathrm{Cr}$, in order to prevent charging.

The rheological behaviour of the material was assessed using a Modular Compact Rheometer (MCR302, Anton Paar, Rivoli, Torino). The measurement setup was composed of a $25 \mathrm{~mm}$ parallel plate system with $1 \mathrm{~mm}$ gap between the plates. Rheological tests were carried out at room temperature, in the steady shear regime. The rheological behaviour of the two different PVDF-DMF solutions was assessed as a function of the shear rate from 0.01 to $100 \mathrm{~s}^{-1}$.

FT-IR characterizations were performed as described in References [12-14]. In particular, FT-IR measurements were carried out in the $4000-600 \mathrm{~cm}^{-1}$ range with a resolution of $1 \mathrm{~cm}^{-1}$ (Bruker Billerica, MA, USA).

The piezoelectric coefficient $d_{33}$ was measured through the PFM technique using a commercial Dimension Icon AFM (Bruker-Veeco, Billerica, MA, USA) $[13,15]$. Following the procedure described in Reference [12], we scanned three different areas $(1 \times 1) \mu \mathrm{m}^{2}$ in size on each sample, with $256 \times 256$ acquisition points per scanning area, and with a scan rate of $0.5 \mathrm{~Hz}$, in order to obtain an estimate of the average value of $d_{33}$, which is representative of the piezoresponse of the sample at macroscale.

The procedure applied for PFM characterization can be summarized as follows:

(i) At first, a calibration measurement of a reference periodically poled lithium niobate (PPLN, Bruker Billerica, MA, USA) sample, with a known value of the piezoresponse coefficient $\left(d_{33 \text { PPLN }}\right)$, is conducted over the amplitude voltage range from $2 \mathrm{~V}$ to $10 \mathrm{~V}$;

(ii) Then, the sample under investigation is tested, and the amplitude of the PFM signal $V_{\text {PVDF }}$ is measured as a function of the applied voltage $V_{a c}$ in the selected range;

(iii) The PPLN is measured again and the new vertical piezoresponse force microscopy signals (VPFM), for each value of the applied voltage $V_{a c}$, are compared with the corresponding values measured during the first calibration cycle; 
(iv) If the difference between the two piezoelectric calibration signals is less than $20 \%$, the measurement of the sample under test is considered reliable;

(v) Next, the calibration factor $\xi=m_{\text {PPLN }} / d_{33 \text { PPLN }}$ (in which $m_{\text {PPLN }}$ is the slope of the straight line of the amplitude of the VPFM signal vs. $V_{a c}$, and $d_{33 \text { PPLN }}$ is the known piezoelectric coefficient of the PPLN) is evaluated, averaging over the two calibration measurements;

(vi) The $d_{33}$ value of the sample under test is finally calculated, using the following expression:

$$
d_{33}=m=\frac{V_{\mathrm{PVDF}}}{\xi V_{\mathrm{ac}}}
$$

in which $V_{\text {PVDF }}$ is the vertical displacement of the sample under test.

Once the PFM measurements were performed in each selected areas of the sample, we estimated the average PFM response as:

$$
\left\langle d_{33}\right\rangle=\left(d_{33}^{1}+d_{33}^{2}+d_{33}^{3}\right) / 3,
$$

and the highest PFM response as:

$$
d_{33 \max }=\max \left\{d_{33}^{1}, d_{33}^{2}, d_{33}^{3}\right\} .
$$

The standard deviation of $\left\langle d_{33}\right\rangle$ is representative of the uniformity of the piezoresponse of the sample.

\section{Results}

\subsection{Rheological Characterization}

The rheological analysis showed a Newtonian behavior of the produced polymer-DMF solutions for both PVDF concentrations of $20 \mathrm{wt} . \%$ and $30 \mathrm{wt} . \%$ The increase in the PVDF to solvent ratio produced an increase in viscosity of the solution, regardless of the shear rate, as shown in Figure 1. Moreover, it can be noticed that the material retains the Newtonian behavior even for the highest polymer concentration. The viscosity of the solution with a polymer concentration of $20 \mathrm{wt} . \%$ is $\eta_{20 \%}=0.79 \mathrm{~Pa} \cdot \mathrm{s}$, while it shows an almost five-fold increase up to the value of $\eta_{30 \%}=3.86 \mathrm{~Pa} \cdot \mathrm{s}$ for a PVDF content of $30 \mathrm{wt} . \%$.

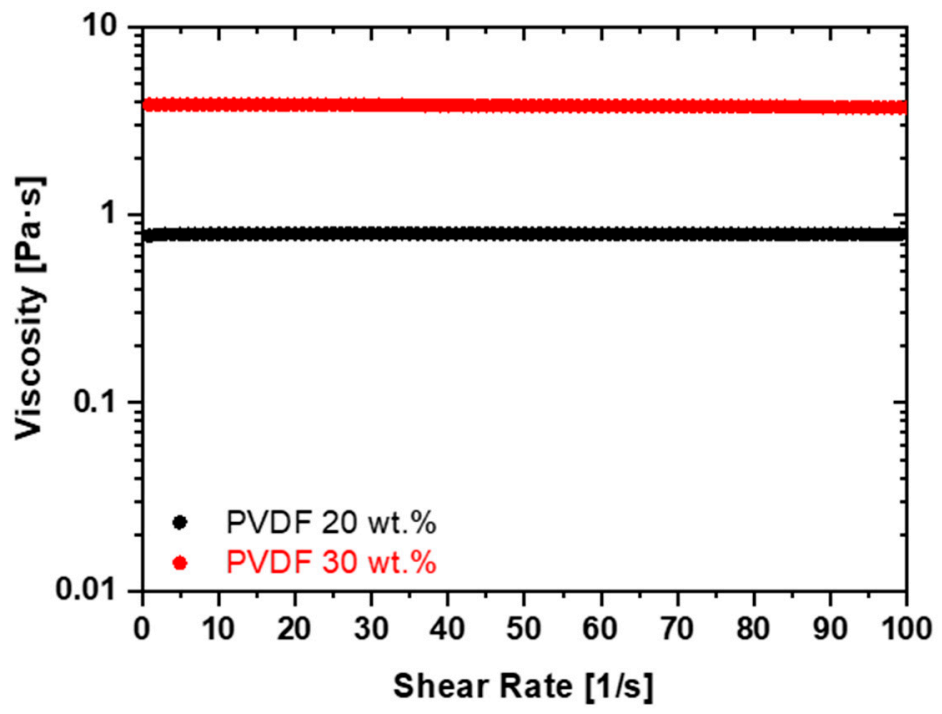

Figure 1. Viscosity curves of PVDF/DMF ( $N, N$ dimethylformamide) solutions with different polymer concentrations. 
On the basis of the rheological characterizations, considering the high values of the spin-coater rotation speed (either $2500 \mathrm{rpm}$ or $7500 \mathrm{rpm}$ ), the relatively short deposition time of $10 \mathrm{~s}$ and the low volatility of DMF at room temperature (i.e., $0.49 \mathrm{kPa}$ ), we can assume that the solvent evaporation is negligible during deposition and that the viscosity of the solution is nearly constant. Therefore, the film thickness $h$ can be estimated using the following expression, according to Reference [16]:

$$
h=\frac{h_{0}}{\sqrt{1+\frac{4 \rho \omega^{2} h_{0}^{2} t}{3 \eta}}}
$$

in which $\omega$ is the spin-coater angular velocity, $t$ is the deposition time, $\eta$ and $\rho$ are the dynamic viscosity and density of the polymer solution, respectively, $h_{0}$ is the initial thickness of the film after the starting transient, i.e., when the plate has reached a constant angular speed. The value of $h_{0}$ can be estimated experimentally, and it is mainly affected by the wettability of the substrate, by the PVDF-DMF solution viscosity, by the initial angular acceleration of the plate providing a twisting force to the polymer. On the basis of previous studies $[10,11]$ and using Equation (4), we can estimate that for the $20 \mathrm{wt} . \%$ PVDF film, $h_{0}$ is in the range 8-20 $\mu \mathrm{m}$, whereas for the $30 \mathrm{wt} . \%$ PVDF film it is in the range $40-70$ $\mu \mathrm{m}$. The density of the solution, $\rho$, is calculated using the following expression: $\rho=M_{\text {solution }} / V_{\text {solution }}$ where $M_{\text {solution }}=m_{P V D F}+m_{D M F}$, being $m_{P V D F}$ and $m_{D M F}$ the measured masses of the PVDF and DMF respectively, and $V_{\text {solution }}=m_{P V D F} / \rho_{P V D F}+m_{D M F} / \rho_{D M F}$, considering the density of PVDF $\rho_{P V D F}=1.78 \mathrm{~g} / \mathrm{cm}^{3}$ and the one of DMF $\rho_{D M F}=0.944 \mathrm{~g} / \mathrm{cm}^{3}$. The calculated densities are: $\rho_{20} \%=1.024$ $\mathrm{g} / \mathrm{cm}^{3}$ and $\rho_{30 \%}=1.060 \mathrm{~g} / \mathrm{cm}^{3}$. Therefore, from Equation (4) and using the measured values of viscosity, we estimate the thickness of the samples, as reported in Table 2. The estimated thickness values are in good agreement with experimentally-measured ones, obtained with a commercial micrometer (see Table 2). The good agreement guarantees the accuracy of the evaluation of the solution's density used in Equation (4).

Table 2. Measured and estimated thickness for the produced samples.

\begin{tabular}{ccccc}
\hline Sample & $\begin{array}{c}\text { PVDF Concentr. } \\
(\mathbf{w t .} \%)\end{array}$ & $\begin{array}{c}\text { Spinning Speed } \\
(\mathbf{r p m})\end{array}$ & $\begin{array}{c}\text { Measured } \\
\text { Thickness } \\
(\boldsymbol{\mu m})\end{array}$ & $\begin{array}{c}\text { Estimated Thickness } \\
\text { from Equation }(\mathbf{4}) \\
(\boldsymbol{\mu m})\end{array}$ \\
\hline $\mathrm{A} / \mathrm{B} / \mathrm{C}$ & 20 & 7500 & $7.7 \pm 1.5$ & 7 \\
$\mathrm{D} / \mathrm{E} / \mathrm{F}$ & 20 & 2500 & $17 \pm 3.0$ & 16 \\
$\mathrm{G} / \mathrm{H} / \mathrm{I}$ & 30 & 7500 & $20 \pm 2.0$ & 18 \\
$\mathrm{~L} / \mathrm{M} / \mathrm{N}$ & 30 & 2500 & $46.0 \pm 2.0$ & 45 \\
\hline
\end{tabular}

\subsection{Surface Morphology}

Figures 2 and 3 show FE-SEM micrographs at low (10 kX) and high ( $25 \mathrm{kX}$ or $50 \mathrm{kX})$ magnifications of the samples listed in Table 1 and produced with a concentration of PVDF in the solvent of $20 \mathrm{wt} . \%$ and the $30 \mathrm{wt} . \%$, respectively.

The produced samples are generally characterized by a porous spherulitic structure, which is originated by solvent evaporation. Size and morphology of the spherulites observed over the surface of the samples are significantly influenced by both spin-coating rotation speed and quenching process.

In particular, it was observed that among the samples produced with the $20 \mathrm{wt} . \%$ of PVDF (Figure 2), the ones deposited with an angular speed of $7500 \mathrm{rpm}$ are characterized by a high porosity; spherulites over the surface of these film are evident only in sample A, which was quenched in water, at room temperature. This suggests that spherulites are mainly produced by the effect of electrostatic interaction with the polar water molecules than by the spinning.

As regards the films produced with a PVDF content of $30 \mathrm{wt} . \%$ (Figure 3), we noticed that they are all characterized by a spherulitic structure. From the SEM images, we estimated the mean value of the spherulite diameter using an image processing software (ImageJ @), National Institute of Health, 
Bethesda, MD, USA) and averaging the diameters of ten different spherulites. The obtained values, reported in Table 1 and demonstrated in this article, show spherulites have minimum dimensions for the samples I and $\mathrm{N}$, which have been quenched in liquid $\mathrm{N}_{2}$ at $77 \mathrm{~K}$. We also observed that samples produced at a lower angular speed ( $2500 \mathrm{rpm}$ ) and quenched at lower temperature (i.e., $253 \mathrm{~K}$ and $77 \mathrm{~K}$ ) are characterized by a more evident stretching and alignment of the polymer chain along the substrate than the ones produced at higher speed (7500 rpm). In particular, the length of the polymeric filaments is limited to $\sim 1 \mu \mathrm{m}$ in samples $\mathrm{H}$ and $\mathrm{I}$, produced at $7500 \mathrm{rpm}$; whereas, they reach several microns in samples $\mathrm{M}$ and $\mathrm{N}$, produced at $2500 \mathrm{rpm}$. Moreover, the averaged diameter of such polymeric filaments is around $300-400 \mathrm{~nm}$ in sample $\mathrm{M}$ and it reduces to $100-200 \mathrm{~nm}$ in sample $\mathrm{N}$ quenched in liquid nitrogen at $77 \mathrm{~K}$.
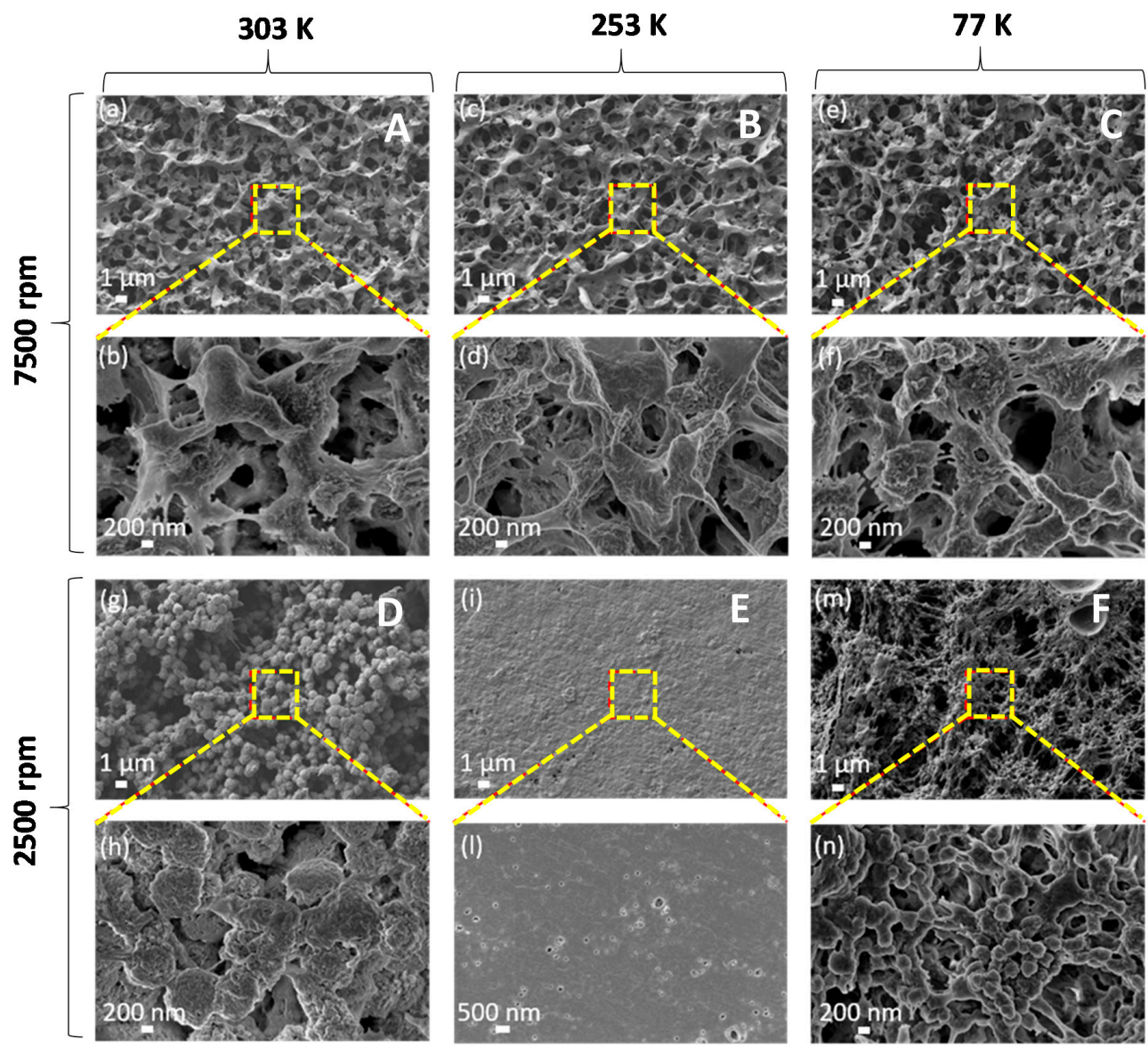

Figure 2. FE-SEM low-magnification $(\mathbf{a}, \mathbf{c}, \mathbf{e}, \mathbf{g}, \mathbf{i}, \mathbf{m})$ and high-magnification $(\mathbf{b}, \mathbf{d}, \mathbf{f}, \mathbf{h}, \mathbf{l}, \mathbf{n})$ micrographs of PVDF films with polymer content of $20 \mathrm{wt}$ \%., produced at spin-coater rotation speed of $7500 \mathrm{rpm}$ (samples A, B, C) or $2500 \mathrm{rpm}$ (samples D, E, F), and quenched at $303 \mathrm{~K}$ in water (samples A, D) or at $253 \mathrm{~K}$ in water and glycerol (samples B, E) or at $77 \mathrm{~K}$ in liquid nitrogen (samples C, F).

\subsection{FT-IR Analysis}

The results of FTIR characterizations are reported in Figure 4a,b. All produced samples exhibit the presence of the $\beta$-phase, as highlighted from the characteristic peaks at $840 \mathrm{~cm}^{-1}$ and at $1275 \mathrm{~cm}^{-1}$. The $\alpha$-phase is generally present in a low amount, as underlined from the relative amplitude of the characteristic peaks, located in the range between 1423 and $763 \mathrm{~cm}^{-1}$ [17-19]. The presence of the $\gamma$-phase is demonstrated from the presence of the peak at $1234 \mathrm{~cm}^{-1}$. 

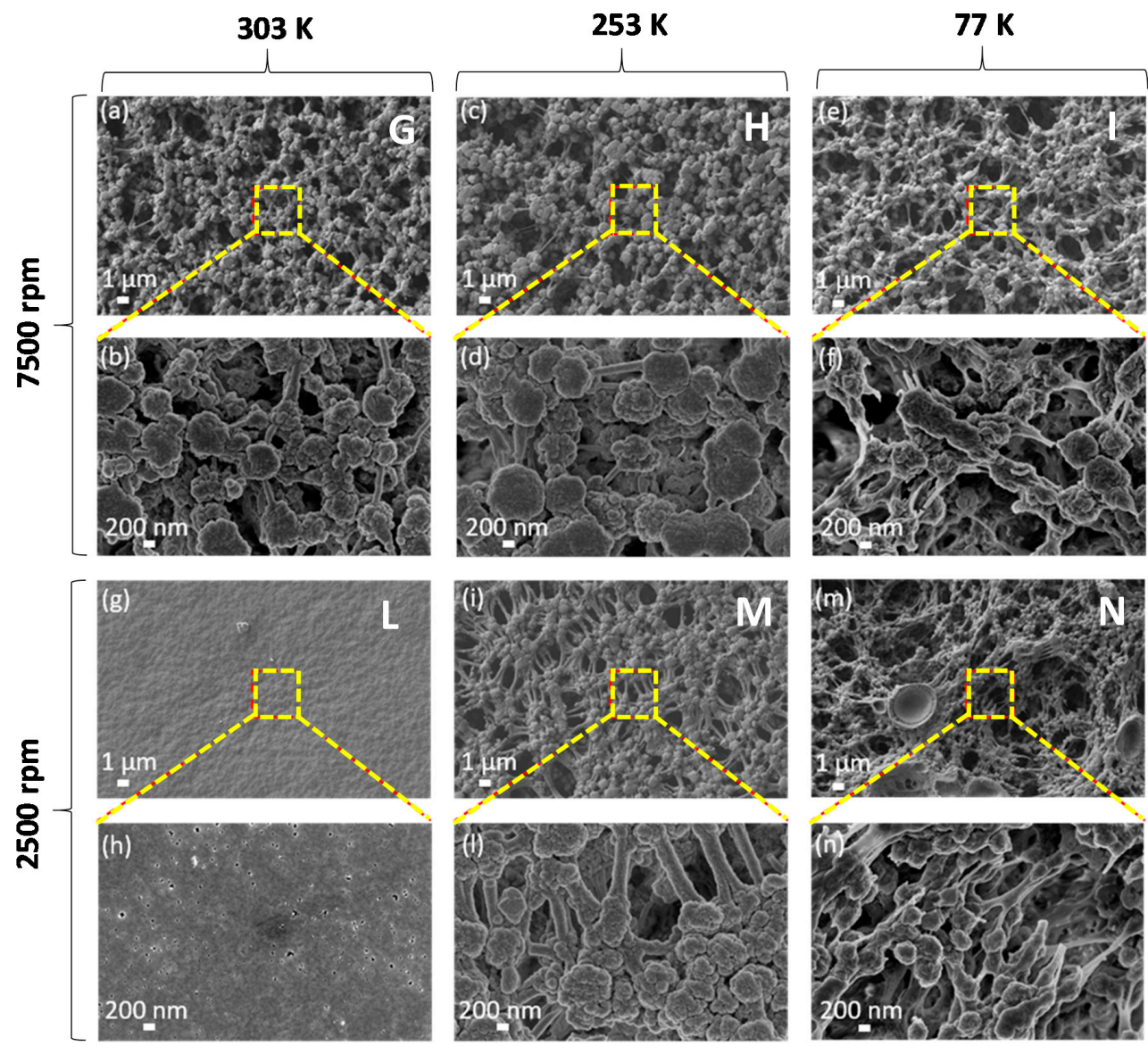

Figure 3. FE-SEM low-magnification (a,c,e,g,i,m) and high-magnification $(\mathbf{b}, \mathbf{d}, \mathbf{f}, \mathbf{h}, \mathbf{l}, \mathbf{n})$ micrographs of PVDF films with polymer content of $30 \mathrm{wt}$.\%., produced at spin-coater rotation speed of $7500 \mathrm{rpm}$ (samples G, H, I) or $2500 \mathrm{rpm}$ (samples L, M, N), and quenched at $303 \mathrm{~K}$ in water (samples G, L) or at $253 \mathrm{~K}$ in water and glycerol (samples $\mathrm{H}, \mathrm{M}$ ) or at $77 \mathrm{~K}$ in liquid nitrogen (samples I, N).

The relative volume fraction of the $\beta$-phase $F(\beta)$ of the produced samples was estimated from the values $A_{\alpha}$ and $A_{\beta}$ of the absorbance at the wavelengths $\left(763\right.$ and $840 \mathrm{~cm}^{-1}$ ) associated to the main peaks of the $\alpha$-and $\beta$-phases, respectively, using the following formula [20]:

$$
F(\beta)=\frac{A_{\beta}}{\left(K_{\beta} / K_{\alpha}\right) A_{\alpha}+A_{\beta}}
$$

in which it is assumed that the ratio between the absorption coefficients of the $\alpha$ - and $\beta$-phases is $K_{\beta} / K_{\alpha} \sim 1.3$.

The obtained values of $F(\beta)$ are reported in Table 1. The highest value of $F(\beta)$ (i.e., $97.82 \%$ ) was observed in sample $\mathrm{N}$, which was produced dissolving $30 \mathrm{wt} . \%$ of PVDF, using a quenching temperature of $77 \mathrm{~K}$ and a spinning speed of $2500 \mathrm{rpm}$. It can be noticed that the obtained values are comparable to those reported in the literature for PVDF thin films obtained using a phase inversion technique through spin-coating and quenching $[2,10,11]$.

\subsection{PFM Characterization}

The morphological maps and the vertical PFM signals over a scan area $(1 \times 1) \mu \mathrm{m}^{2}$ are reported in Figures 5 and 6 for all produced samples of Table 1 . The average value $\left\langle d_{33}\right\rangle$ was obtained, according to the procedure described in Section 2, from the slope of the linear fitting of the VPFM as a function of $V_{a c}$ (Figure 7). The values of $\left\langle d_{33}\right\rangle$, with the corresponding standard deviations and of the maximum value of $d_{33}$, are also included in Table 1 and reported in Figure 8 as a function of $F(\beta)[12,14]$. 

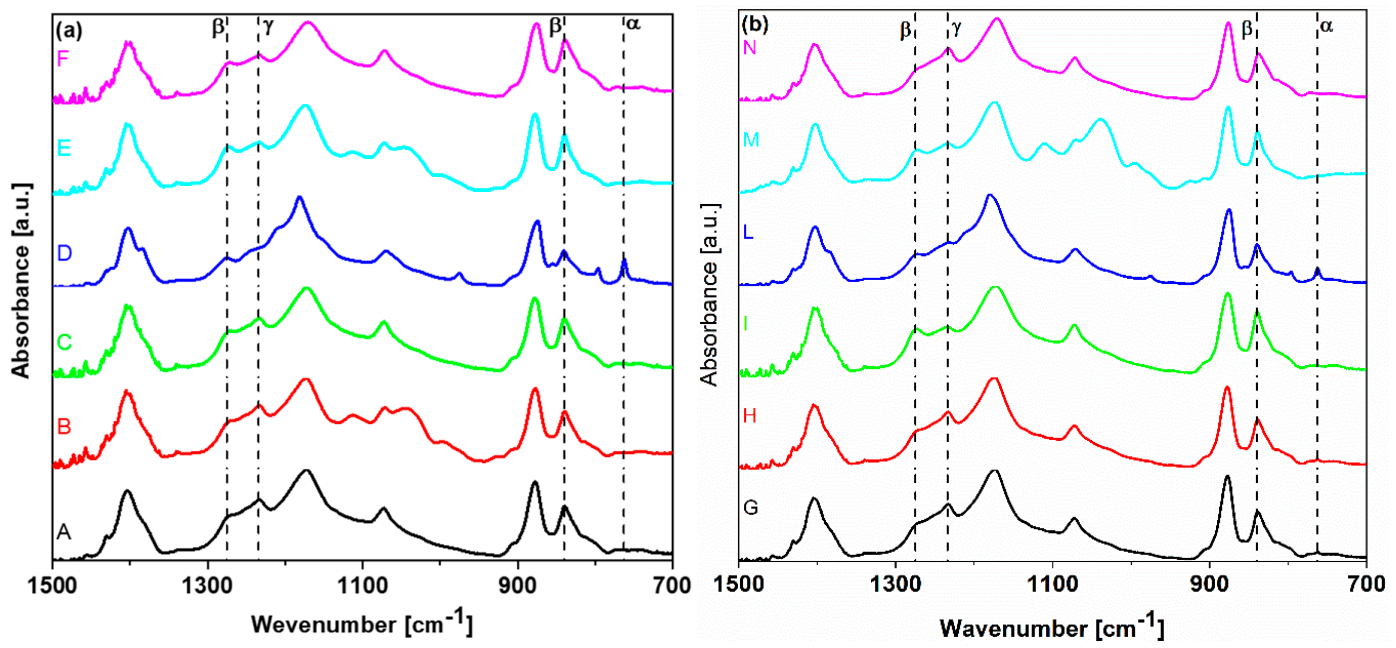

Figure 4. FT-IR spectra of 12 PVDF solutions.
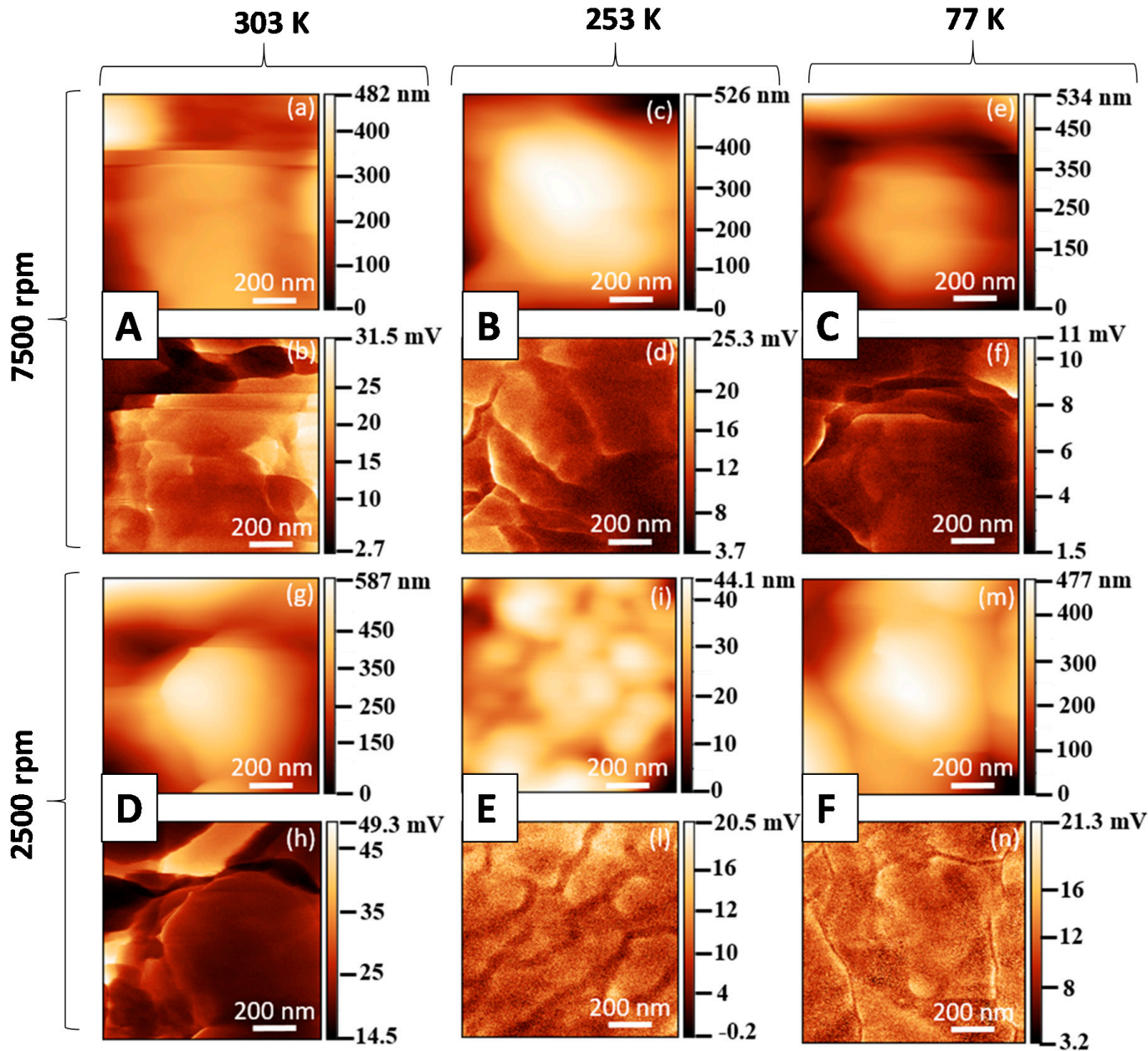

Figure 5. Morphological maps and Piezoresponse Force Microscopy (PFM) signals at $\mathrm{V}_{\mathrm{ac}}=10 \mathrm{~V}$ and at $15 \mathrm{kHz}$ for PVDF at $20 \mathrm{wt}$.\% produced using different temperatures and spinning speeds: (a,b) Sample A, (c,d) sample B, (e,f) sample $C,(\mathbf{g}, \mathbf{h})$ sample $D,(\mathbf{i}, \mathbf{l})$ sample $E,(\mathbf{m}, \mathbf{n})$ sample $F$. 

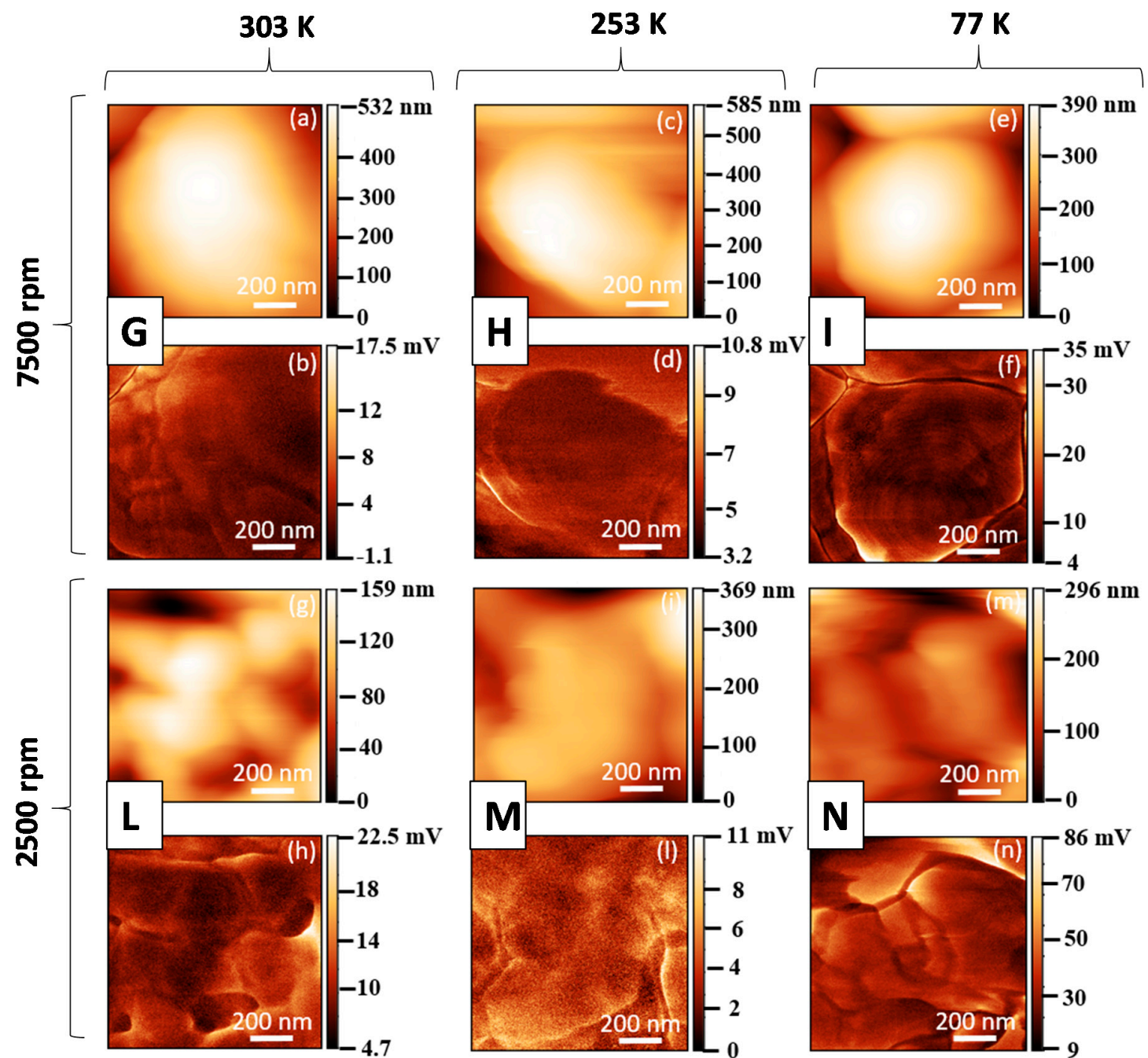

Figure 6. Morphological maps and PFM signals at $\mathrm{V}_{\mathrm{ac}}=10 \mathrm{~V}$ and at $15 \mathrm{kHz}$ for PVDF at $30 \mathrm{wt} . \%$ produced at different temperatures and spinning speeds: (a,b) Sample G, (c,d) sample $H,(\mathbf{e}, \mathbf{f})$ sample I, (g,h) sample L, (i,l) sample M, (m,n) sample N.
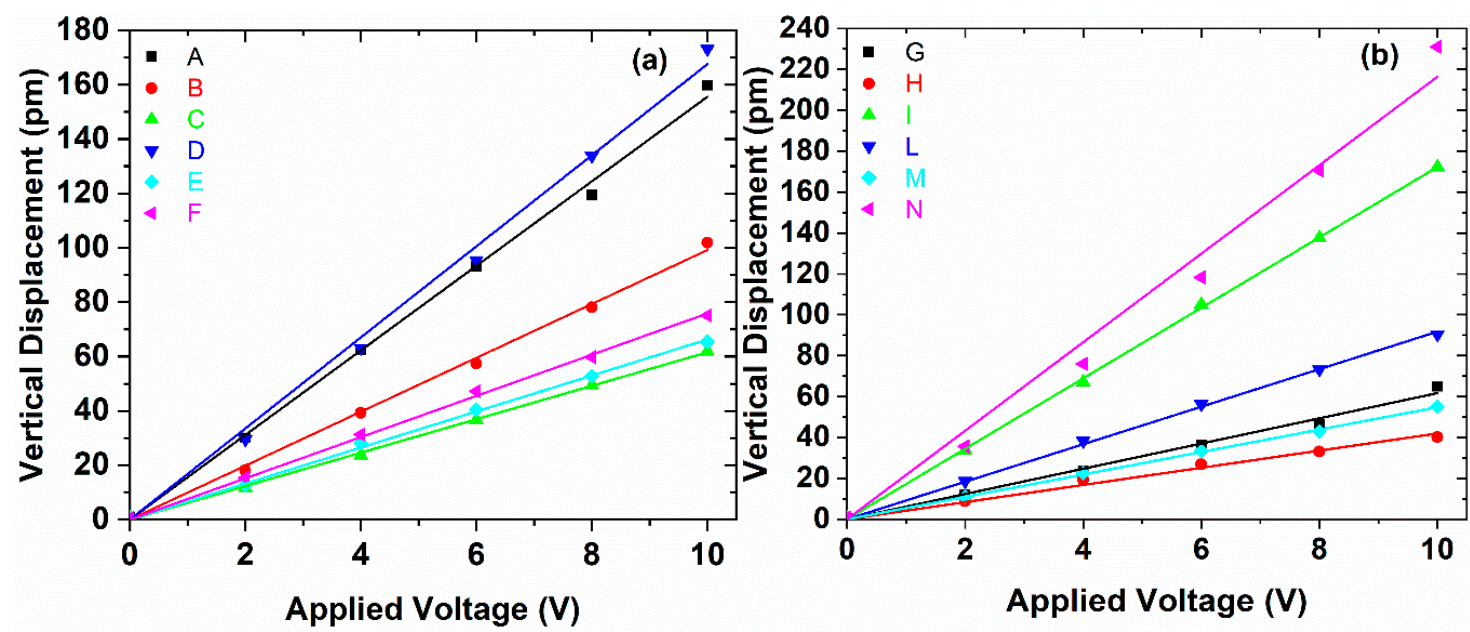

Figure 7. The average amplitude of the vertical displacement, measured through PFM as a function of the applied voltage $V_{a c}$ : For the PVDF samples at $20 \mathrm{wt} . \%$ at different temperatures and spinning speeds (a) and for the PVDF samples at $30 \mathrm{wt} . \%$ at different temperature and spinning speed (b). 


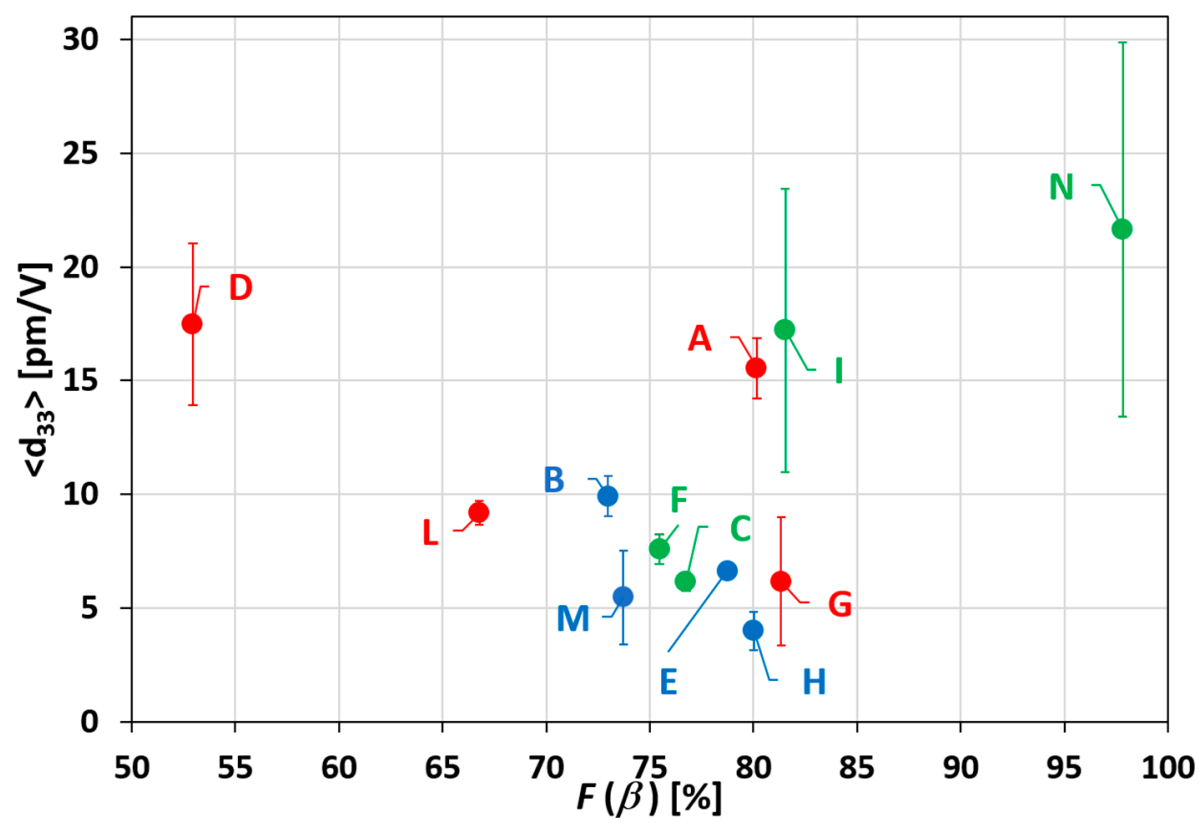

Figure 8. Results of the averaged $d_{33}$ as a function of $F(\beta)$ of all produced samples, with standard deviation.

It is interesting to observe that the samples produced at $253 \mathrm{~K}$ show lower piezoelectric coefficient, although the $F(\beta)$ is always higher than $70 \%$. Furthermore, among the samples quenched at $303 \mathrm{~K}$ (i.e., A, D, G, L), the ones containing the lower polymer concentration (i.e., samples A and D), show a better piezoresponse. On the contrary, if the samples are quenched at $77 \mathrm{~K}$, the higher piezoelectric response is obtained when the highest polymer concentration (30 wt.\%) is used samples (L, N). The highest value of $\left\langle d_{33}\right\rangle=(21.64 \pm 8.23) \mathrm{pm} / \mathrm{V}$ and of $d_{33 \max }=29.87 \mathrm{pm} / \mathrm{V}$ was measured for the sample $\mathrm{N}$, which is also characterized by the highest value of $F(\beta)=97.82 \%$.

\section{Discussion}

Electroactive phase formation in PVDF films produced through spin-coating and quenching has been investigated in order to assess the main factors that affect phase inversion. In fact, several studies highlighted that $\beta$-phase formation in PVDF films is the result of the action of several factors, such as $[2,10,11]$ : (i) The mechanical stretching of the polymeric chains induced during spin-coating; (ii) the thermal gradient acting on the deposited film during quenching in a water bath below $0{ }^{\circ} \mathrm{C}$; (iii) the electrostatic interaction between the $\mathrm{CF}_{2}-\mathrm{CH}_{2}$ groups of PVDF and polar molecules of water.

In this study, we demonstrated that the aforementioned three factors do not always contribute effectively to the enhancement of the piezoelectric properties of the material. Moreover, depending on the selected spin-coating condition, PVDF concentration in the initial DMF solution, and expected thickness of the film, it is possible to choose the proper quenching treatment enabling to achieve the best enhancement of the piezoelectric properties of the material.

For this purpose, we considered three different quenching conditions:

(1) Quenching at room temperature in water: The electrostatic interaction with the polar molecules of $\mathrm{H}_{2} \mathrm{O}$ is the dominant polarization factor during polymer crystallization; 
(2) Quenching at $253 \mathrm{~K}$ in a water/glycerol solution: The temperature gradient across the sample combined with the electrostatic interaction with water molecules are the main polarization factors acting during polymer crystallization;

(3) Quenching at $77 \mathrm{~K}$ in liquid nitrogen: Neither the temperature gradient, nor the electrostatic interaction, are present. In this case, quenching has only the function of producing an instantaneous crystallization of the polymer, so that mechanical relaxation after spin-coating deposition is inhibited.

Therefore, from the combined analysis of the morphological, FTIR and PFM investigations, we can draw the following conclusions.

The best stretching and alignment of the polymeric chains along the substrate is obtained producing the film by spin-coating at a lower angular speed (i.e., $2500 \mathrm{rpm}$ ). During spin-coating deposition, due to the very different molecular mass of fluorine and hydrogen, the centrifugal force induces orientation of the polar groups of $\mathrm{CF}_{2}$ and $\mathrm{CH}_{2}$ in PVDF. Therefore, the rapid freezing of the PVDF film at very low temperature in liquid nitrogen, which is an apolar molecule, produces an instantaneous crystallization of the material, thus preventing elastic relaxation of the polymeric chains. This phenomenon is the dominant one, especially for the case of solutions with a higher viscosity, containing $30 \mathrm{wt} . \%$ of PVDF. This is confirmed by the high value of $\beta$-phase content, by the high value of measured piezoresponse coefficient, and by the formation of spherulites over the sample surface, interconnected by polymeric filaments that have a diameter below $200 \mathrm{~nm}$, and lengths exceeding a few microns.

In the case of a more dilute solution, made with PDVF solution of $20 \mathrm{wt} . \%$, the effect of mechanical stretching during spin-coating is limited, due to the low viscosity of the polymeric solution. Actually, samples with $20 \mathrm{wt} . \%$ of PVDF quenched at $77 \mathrm{~K}$ in liquid nitrogen, were characterized by a low piezoresponse coefficient. This confirms that $\beta$-phase crystallization is produced essentially through the electrostatic interaction of PVDF polar groups with polar molecules of water, occurring during quenching in a water bath at room temperature $(303 \mathrm{~K})$. In fact, the mechanism that is believed to be responsible for the increase of the piezoelectric coefficient in the sample at $20 \mathrm{wt} \%$ of PVDF quenched at $303 \mathrm{~K}$, is the formation of hydrogen bonds between the $\mathrm{O}-\mathrm{H}$ groups of water with $\mathrm{C}-\mathrm{H}$ groups of PVDF. The formation of these hydrogen bonds induce the PVDF molecules on the water to be oriented with C-F groups downwards to water, as described in References $[10,21]$. The contextual action of a thermal gradient originated by cooling in a bath of water and glycerol (i.e., quenching at $253 \mathrm{~K}$ ) is not constructive with respect to the effect of electrostatic interaction. In fact, we observed that the highest value of piezoresponse was measured in samples at $20 \mathrm{wt} . \%$ of PVDF, quenched in water at room temperature. These samples are also characterized by spherulitic structures, due to the solid-liquid phase separation that occurs during the crystallization phase [12-14,22], with an average spherulite diameter in the range 700-800 nm. Differently, from the samples produced with PVDF at $30 \mathrm{wt} . \%$, the formation of polymer filaments is not observed over the film surface: This confirms that the effect of mechanical stretching produced by spin-coating deposition is not the dominant factor in the enhancement of the piezoresponse.

From the histogram of Figure 9, it is evident that the increase of the piezoresponse of the material is obtained through quenching at the highest temperature of $303 \mathrm{~K}$ of the film produced with the less concentrated PVDF solution. On the contrary, it is achieved through quenching at the lowest temperature of $77 \mathrm{~K}$ in the case of films produced by solution with the highest concentration of PVDF. 


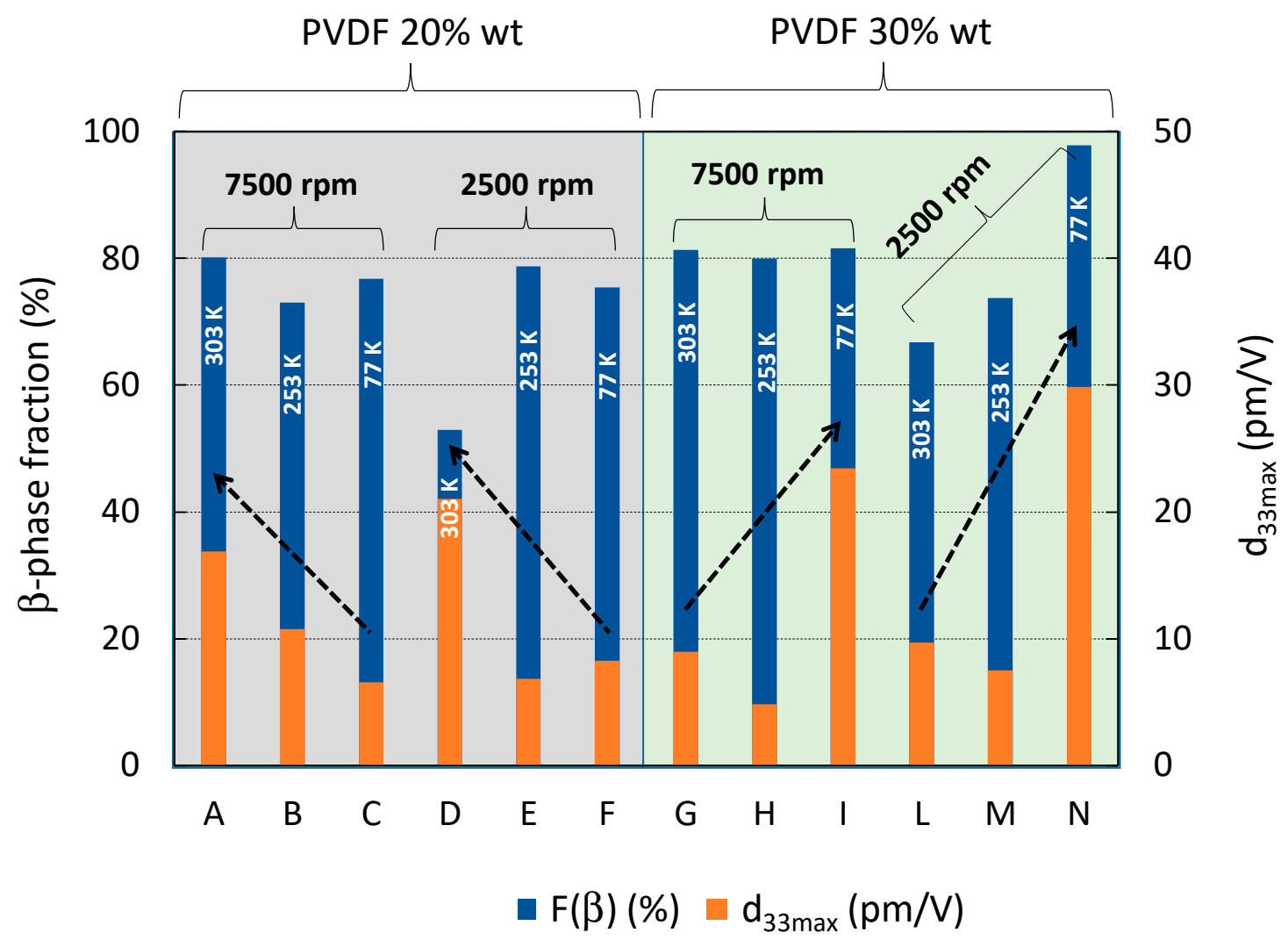

Figure 9. $\beta$-phase relative volume fraction and maximum measured piezoresponse coefficient of all produced samples.

\section{Conclusions}

In this paper, we demonstrated that the process that enables to obtain films with the highest $\beta$-phase and piezoresponse coefficient depends on the initial concentration of PVDF over DMF. In fact, the viscosity of the polymer solution plays a crucial role in the orientation of the polymer chains, due to mechanical stretching induced by spinning.

FT-IR and PFM data, in some cases, do not have the same trend as a function of the quenching temperature. This is due to the fact that although the FT-IR measurements give us information about the amount of $\beta$-phase in the sample, nevertheless they do not give us any information about the orientation of the polymeric chains. In any case, it is important to underline that the highest $d_{33}$ is associated with the highest amount of $F(\beta)$.

The samples quenched at $303 \mathrm{~K}$ showed the best $d_{33}$ among the samples produced with $20 \mathrm{wt} . \%$ of PVDF, indicating that the dominant factor in phase inversion is the electrostatic interaction between the polar groups of PVDF and water molecules. For the samples with a polymer/solvent mass ratio of $30 \mathrm{wt} . \%$, the best $d_{33}$ is obtained using a quenching temperature of $77 \mathrm{~K}$, indicating a better orientation and alignment of the polymer chains, which are frozen using liquid $\mathrm{N}_{2}$.

In this work, the combination of the deposition of thin films through the spin process, and the phase inversion through a quenching process, gave us a considerable enhancement of the $d_{33}$ if compared with our previous studies [12-14,23], as well as with works reported in the literature for PVDF composites and other piezoelectric materials [7-10,24-29].

The combination of spin-coating and phase inversion opensspi a new way to realize highly efficient piezoelectric PVDF thin films with an enhanced $d_{33}$, without the need of electrical poling or mechanical stretching, suitable to realize devices for energy harvesting and wearable sensors. 
Author Contributions: Conceptualization and Methodology: M.F., A.T., M.S.S.; Sample production: D.C., M.F.; Rheology measurements: F.M.; SEM characterizations: G.D.B.; FTIR analysis: F.S.; PFM measurements, M.F.; Data Curation: M.F.; Writing_-Original Draft Preparation and Editing: M.F., A.T., M.S.S.; Review: All; Supervision: M.S.S.; Project Administration: M.S.S.; Funding Acquisition: M.S.S.

Funding: This research was funded by INAIL SENSE RISC [ID 10/2018] project grand number [B56C18004200005].

Conflicts of Interest: The authors declare no conflict of interest.

\section{References}

1. Fortunato, M.; Rinaldi, A.; Tamburrano, A.; De Bellis, G.; Dikonimos, T.; Lisi, N.; Sarto, M.S. Graphene-Gold Electrodes for Flexible Nanogenerators Based on Porous Piezoelectric PVDF Films. In Proceedings of the 18th International Conference on Nanotechnology (IEEE-NANO), Cork, Ireland, 23-26 July 2018; pp. 1-4.

2. Cardoso, V.F.; Minas, G.; Costa, C.M.; Tavares, C.J.; Lanceros-Mendez, S. Micro and nanofilms of poly(vinylidene fluoride) with controlled thickness, morphology and electroactive crystalline phase for sensor and actuator applications. Smart Mater. Struct. 2011, 20, 087002. [CrossRef]

3. Li, J.; Seok SIl Chu, B.; Dogan, F.; Zhang, Q.; Wang, Q. Nanocomposites of Ferroelectric Polymers with TiO2 Nanoparticles Exhibiting Significantly Enhanced Electrical Energy Density. Adv. Mater. 2009, 21, $217-221$. [CrossRef]

4. Granstrom, J.; Feenstra, J.; Sodano, H.A.; Farinholt, K. Energy harvesting from a backpack instrumented with piezoelectric shoulder straps. Smart Mater. Struct. 2007, 16, 1810-1820. [CrossRef]

5. Coster, H.G.L.; Farahani, T.D.; Chilcott, T.C. Production and characterization of piezo-electric membranes. Desalination 2011, 283, 52-57. [CrossRef]

6. Guo, D.; Zeng, F.; Dkhil, B. Ferroelectric Polymer Nanostructures: Fabrication, Structural Characteristics and Performance Under Confinement. J. Nanosci. Nanotechnol. 2014, 14, 2086-2100. [CrossRef]

7. Gomes, J.; Serrado Nunes, J.; Sencadas, V.; Lanceros-Mendez, S. Influence of the $\beta$-phase content and degree of crystallinity on the piezo- and ferroelectric properties of poly(vinylidene fluoride). Smart Mater. Struct. 2010, 19, 065010. [CrossRef]

8. Kang, S.B.; Won, S.H.; Im, M.J.; Kim, C.U.; Park WIl Baik, J.M.; Choi, K.J. Enhanced piezoresponse of highly aligned electrospun poly(vinylidene fluoride) nanofibers. Nanotechnology 2017, 28, 395402. [CrossRef]

9. Ramasundaram, S.; Yoon, S.; Kim, K.J.; Lee, J.S. Direct Preparation of Nanoscale Thin Films of Poly(vinylidene fluoride) Containing $\beta$-Crystalline Phase by Heat-Controlled Spin Coating. Macromol. Chem. Phys. 2008, 209, 2516-2526. [CrossRef]

10. Soin, N.; Boyer, D.; Prashanthi, K.; Sharma, S.; Narasimulu, A.A.; Luo, J.; Shah, T.H.; Siores, E.; Thundat, T. Exclusive self-aligned $\beta$-phase PVDF films with abnormal piezoelectric coefficient prepared via phase inversion. Chem. Commun. 2015, 51, 8257-8260. [CrossRef]

11. Mahale, B.; Bodas, D.; Gangal, S.A. Study of $\beta$-phase development in spin-coated PVDF thick films. Bull. Mater. Sci. 2017, 40, 569-575. [CrossRef]

12. Fortunato, M.; Chandraiahgari, C.; De Bellis, G.; Ballirano, P.; Sarto, F.; Tamburrano, A.; Sarto, M. Piezoelectric Effect and Electroactive Phase Nucleation in Self-Standing Films of Unpoled PVDF Nanocomposite Films. Nanomaterials 2018, 8, 743. [CrossRef]

13. Fortunato, M.; Bidsorkhi, H.C.; De Bellis, G.; Sarto, F.; Sarto, M.S. Piezoelectric response of graphene-filled PVDF nanocomposites through Piezoresponse Force Microscopy (PFM). In Proceedings of the 17th International Conference on Nanotechnology (IEEE-NANO), Pittsburgh, PA, USA, 25-28 July 2017; pp. 125-129.

14. Fortunato, M.; Bidsorkhi, H.C.; Chandraiahgari, C.R.; De Bellis, G.; Sarto, F.; Sarto, M.S. PFM Characterization of PVDF Nanocomposite Films With Enhanced Piezoelectric Response. IEEE Trans. Nanotechnol. 2018, 17, 955-961. [CrossRef]

15. Soergel, E. Piezoresponse force microscopy (PFM). J. Phys. D Appl. Phys. 2011, 44, 464003. [CrossRef]

16. Tyona, M.D. A comprehensive study of spin coating as a thin film deposition technique and spin coating equipment. Adv. Mater. Res. 2013, 2, 181-193. [CrossRef]

17. Mandal, D.; Henkel, K.; Schmeißer, D. The electroactive $\beta$-phase formation in Poly(vinylidene fluoride) by gold nanoparticles doping. Mater. Lett. 2012, 73, 123-125. [CrossRef] 
18. Chandraiahgari, C.R.; De Bellis, G.; Ballirano, P.; Balijepalli, S.K.; Kaciulis, S.; Caneve, L.; Sarto, F.; Sarto, M.S. Synthesis and characterization of $\mathrm{ZnO}$ nanorods with a narrow size distribution. RSC Adv. 2015, 5, 49861-49870. [CrossRef]

19. Garain, S.; Sinha, T.K.; Adhikary, P.; Henkel, K.; Sen, S.; Ram, S.; Sinha, C.; Schmeißer, D.; Mandal, D. Self-Poled Transparent and Flexible UV Light-Emitting Cerium Complex-PVDF Composite: A High-Performance Nanogenerator. ACS Appl. Mater. Interfaces 2015, 7, 1298-1307. [CrossRef]

20. Gregorio, R., Jr.; Cestari, M. Effect of crystallization temperature on the crystalline phase content and morphology of poly(vinylidene fluoride). J. Polym. Sci. Part B Polym. Phys. 1994, 32, 859-870. [CrossRef]

21. Chen, S.; Li, X.; Yao, K.; Tay, F.E.H.; Kumar, A.; Zeng, K. Self-polarized ferroelectric PVDF homopolymer ultra-thin films derived from Langmuir-Blodgett deposition. Polymer 2012, 53, 1404-1408. [CrossRef]

22. Bidsorkhi, H.C.; D'Aloia, A.G.; De Bellis, G.; Proietti, A.; Rinaldi, A.; Fortunato, M.; Ballirano, P.; Bracciale, M.P.; Santarelli, M.L.; Sarto, M.S. Nucleation effect of unmodified graphene nanoplatelets on PVDF/GNP film composites. Mater. Today Commun. 2017, 11, 163-173. [CrossRef]

23. Fortunato, M.; Chandraiahgari, C.R.; De Bellis, G.; Ballirano, P.; Soltani, P.; Kaciulis, S.; Caneve, L.; Sarto, F.; Sarto, M.S. Piezoelectric Thin Films of ZnO-Nanorods/Nanowalls Grown by Chemical Bath Deposition. IEEE Trans. Nanotechnol. 2018, 17, 311-319. [CrossRef]

24. Lopes, A.C.; Costa, C.M.; Tavares, C.J.; Neves, I.C.; Lanceros-Mendez, S. Nucleation of the Electroactive $\gamma$ Phase and Enhancement of the Optical Transparency in Low Filler Content Poly(vinylidene)/Clay Nanocomposites. J. Phys. Chem. C 2011, 115, 18076-18082. [CrossRef]

25. Kim, G.H.; Hong, S.M.; Seo, Y. Piezoelctric properties of poly(vnylidene fluoride) and carbon nanotube blends: $\beta$-phase development. Phys. Chem. Chem. Phys. 2009, 11, 10506-10512. [CrossRef]

26. Bystrov, V.S.; Bdikin, I.K.; Silibin, M.V.; Karpinsky, D.V.; Kopyl, S.A.; Goncalves, G.; Sapronova, A.V.; Kuznetsova, T.; Bystrova, V.V. Graphene/graphene oxide and polyvinylidene fluoride polymer ferroelectric composites for multifunctional applications. Ferroelectrics 2017, 509, 124-142. [CrossRef]

27. Ramadan, K.S.; Sameoto, D.; Evoy, S. A review of piezoelectric polymers as functional materials for electromechanical transducers. Smart Mater. Struct. 2014, 23, 033001. [CrossRef]

28. Scrymgeour, D.A.; Sounart, T.L.; Simmons, N.C.; Hsu, J.W.P. Polarity and piezoelectric response of solution grown zinc oxide nanocrystals on silver. J. Appl. Phys. 2007, 101, 014316. [CrossRef]

29. Wan, C.; Bowen, C.R. Multiscale-structuring of polyvinylidene fluoride for energy harvesting: The impact of molecular-, micro- and macro-structure. J. Mater. Chem. A 2017, 5, 3091-3128. [CrossRef] 